\title{
Noncommutative Phase Space Schrödinger Equation with Minimal Length
}

\author{
H. Hassanabadi, ${ }^{1}$ Z. Molaee, ${ }^{2}$ and S. Zarrinkamar ${ }^{2}$ \\ ${ }^{1}$ Department of Basic Sciences, Shahrood Branch, Islamic Azad University, Shahrood, Iran \\ ${ }^{2}$ Department of Basic Sciences, Garmsar Branch, Islamic Azad University, Garmsar, Iran
}

Correspondence should be addressed to H. Hassanabadi; h.hasanabadi@shahroodut.ac.ir

Received 23 October 2013; Revised 12 December 2013; Accepted 18 December 2013; Published 30 January 2014

Academic Editor: Elias C. Vagenas

Copyright (C) $2014 \mathrm{H}$. Hassanabadi et al. This is an open access article distributed under the Creative Commons Attribution License, which permits unrestricted use, distribution, and reproduction in any medium, provided the original work is properly cited. The publication of this article was funded by SCOAP S $^{3}$

We consider the Schrödinger equation under an external magnetic field in two-dimensional noncommutative phase space with an explicit minimal length relation. The eigenfunctions are reported in terms of the Jacobi polynomials, and the explicit form of energy eigenvalues is reported.

\section{Introduction}

Although the advent of Schrödinger equation dates back to many decades ago [1-3], the interest in the study of the equation has been renewed due to the noncommutative phase space commutations relations and the so-called minimal length (alternatively called generalized) uncertainty relation. The noncommutativity between space-time coordinates was first explained by Snyder [4] and provides us with motivating scenarios in string and M-theories [5, 6] as well as modern cosmology [7-9]. The application of the formulation is not limited to these fields and noncommutative theories are capable of explaining the IR/UV mixing and nonlocality [10], Lorentz violation [11], and new physics at very small scales $[12,13]$. Extensive studies on the incorporation of ordinary quantum mechanics and classical mechanics with the noncommutative relations can be found in [14-16]. On the other hand, the modification of our ordinary uncertainty relation, which is inspired by the string theory, quantum gravity, and doubly special relativity [17-20], has become an appealing case of study in the recent years. In the minimal length formulation, the ordinary Heisenberg relation is modified by an ad hoc term and is written as $[\widehat{X}, \widehat{P}]=i\left(1+\beta \widehat{P}^{2}\right)$, where $\beta$ is a small parameter determined from a fundamental theory such as string theory $[21,22]$. Such a commutation relation corresponds to the following uncertainty relation:
$\Delta \widehat{X} \geq(1 / 2)((1 / \Delta \widehat{P})+\beta \Delta \widehat{P})$, which implies the existence of a minimal length $\Delta \widehat{X}_{0}=\sqrt{\beta}[21,22]$. Recently, the universality of quantum gravity corrections was studied by Das and Vagenas. They indicated the existence of a minimum measurable length and the related generalized uncertainty principle (GUP) [23]. In another interesting paper, Ali et al. considered a GUP consistent with string theory, black hole physics, and doubly special relativity [24].

In the present work, we combine these topics within the framework of nonrelativistic Schrödinger equation. The organization of this work is as follows. In Section 2, we consider the Schrödinger equation in the presence of magnetic field in noncommutative phase space (NCPS). In Section 3, we study this problem with minimal length relations. In Section 4, we consider some special cases of the solutions to check the validity of the results.

\section{The Two-Dimensional Schrödinger Equation in Noncommutative Phase Space}

The free particle Schrödinger equation in two spatial dimensions $(\hbar=c=1)$ reads

$$
\left((\vec{P}-q \vec{A})^{2}-2 M E_{n, m}\right) \psi(x, y)=0,
$$


where $M$ is mass of the particle. Using the operator relations

$$
P_{x}=\frac{1}{i} \frac{d}{d x}, \quad P_{y}=\frac{1}{i} \frac{d}{d y}
$$

and recalling that the vector potential is written as $\vec{A}=$ $(-(B / 2) y,(B / 2) x, 0)$, where $B$ is magnetic field, (1) takes the form

$$
\begin{aligned}
& \left(-\left(P_{x}^{(\mathrm{NC})}+\frac{q B}{2} y^{(\mathrm{NC})}\right)^{2}-\left(P_{y}^{(\mathrm{NC})}-\frac{q B}{2} x^{(\mathrm{NC})}\right)^{2}\right. \\
& \left.+2 M E_{n, m}\right) \psi(x, y)=0 .
\end{aligned}
$$

In NCPS the position operators are satisfied in the following relations [25-27]:

$$
\left[x^{\mu(\mathrm{NC})}, x^{\nu(\mathrm{NC})}\right]=i \theta^{\mu \nu},
$$

where $\theta^{\mu \nu}$ is an antisymmetric tensor and is of space dimension $(L)^{2}$. The other commutation relations appear as

$$
\begin{gathered}
{\left[x^{i(\mathrm{NC})}, x^{j(\mathrm{NC})}\right]=i \theta^{i j}, \quad\left[P^{i(\mathrm{NC})}, P^{j(\mathrm{NC})}\right]=i \eta^{i j},} \\
{\left[x^{i(\mathrm{NC})}, P^{j(\mathrm{NC})}\right]=i \hbar_{\mathrm{eff}} \delta^{i j}}
\end{gathered}
$$

with the effective plank constant being

$$
\begin{gathered}
\hbar_{\mathrm{eff}}=\left(1+\frac{\theta \eta}{4}\right), \\
x^{i(\mathrm{NC})}=\widehat{x}_{i}-\frac{\theta^{i j} \widehat{p}_{j}}{2}, \quad P^{i(\mathrm{NC})}=\widehat{p}_{i}+\frac{\eta^{i j} \widehat{x}_{j}}{2},
\end{gathered}
$$

where $\theta^{i j}=\varepsilon^{i j k} \theta_{k}, \theta_{k}=(0,0, \theta), \eta^{i j}=\varepsilon^{i j k} \eta_{k}$, and $\eta_{k}=(0,0, \eta)$ are the noncommutative parameters. The NCPS parameters are related to the commutative space parameters via

$$
\begin{array}{ll}
P_{x}^{(\mathrm{NC})}=\widehat{P}_{x}^{(\mathrm{C})}+\frac{\eta \widehat{y}^{(\mathrm{C})}}{2}, & x^{(\mathrm{NC})}=\widehat{x}^{(\mathrm{C})}-\frac{\theta \widehat{P}_{y}^{(\mathrm{C})}}{2}, \\
P_{y}^{(\mathrm{NC})}=\widehat{P}_{y}^{(\mathrm{C})}-\frac{\eta \widehat{x}^{(\mathrm{C})}}{2}, & y^{(\mathrm{NC})}=\widehat{y}^{(\mathrm{C})}+\frac{\theta \widehat{P}_{x}^{(\mathrm{C})}}{2} .
\end{array}
$$

By inserting (7) into (3), we obtain the equation in commutative space as

$$
\begin{aligned}
& \left\{2 M E_{n, m}-\left(1+\frac{q B \theta}{4}\right)^{2}\left(\widehat{P}_{x}^{(\mathrm{C}) 2}+\widehat{P}_{y}^{(\mathrm{C}) 2}\right)\right. \\
& -\left(\frac{\eta}{2}+\frac{q B}{2}\right)^{2}\left(\widehat{x}^{(\mathrm{C}) 2}+\widehat{y}^{(\mathrm{C}) 2}\right)+\left(\frac{\eta}{2}+\frac{q B}{2}\right) \\
& \left.\times\left(1+\frac{q B \theta}{4}\right) L_{z}\right\} \psi_{n, m}(\hat{x}, \hat{y})=0,
\end{aligned}
$$

in which $L_{z}=i(x(\partial / \partial y)-y(\partial / \partial x))$ is angular momentum.

\section{The Problem with a Minimal Length}

For the sake of simplicity, we bring the problem into the momentum space. Recalling that $\widehat{x}_{i}=i \hbar\left(1+\beta P^{2}\right) \partial / \partial P_{i}$, $\widehat{P}_{i}=P_{i}[28]$,

$$
\begin{aligned}
\hat{y}=i\left(1+\beta P^{2}\right) \frac{\partial}{\partial P_{y}}, & \widehat{x}=i\left(1+\beta P^{2}\right) \frac{\partial}{\partial P_{x}}, \\
\widehat{P}_{x}=P_{x}, & \widehat{P}_{y}=P_{y},
\end{aligned}
$$

as well as writing

$$
\widehat{P}_{y}^{(\mathrm{C})}=P \sin \varphi, \quad \widehat{P}_{x}^{(\mathrm{C})}=P \cos \varphi, \quad P^{2}=\widehat{P}_{x}^{(\mathrm{C}) 2}+\widehat{P}_{y}^{(\mathrm{C}) 2},
$$

and $\psi_{n, m}=\psi_{n, m}(P, \varphi)=U_{n}^{\prime}(P) Q_{m}(\varphi)$, where $Q_{m}(\varphi)=e^{i m \varphi}$, the Schrödinger equation appears as

$$
\begin{gathered}
\left\{\frac{d^{2}}{d P^{2}}+\frac{1}{P} \frac{d}{d P}+\frac{-m^{2}}{P^{2}}+\frac{2 M E_{n, m}}{((\eta / 2)+(q B / 2))^{2}\left(1+\beta P^{2}\right)^{2}}\right. \\
-\frac{\beta}{1+\beta P^{2}}-\frac{(1+(q B \theta / 4))^{2} P^{2}}{((\eta / 2)+(q B / 2))^{2}\left(1+\beta P^{2}\right)^{2}} \\
\left.+\frac{2 \beta P}{1+\beta P^{2}} \frac{d}{d P}+\frac{(1+(q B \theta / 4)) m}{((\eta / 2)+(q B / 2))\left(1+\beta P^{2}\right)}\right\} U_{n}^{\prime}=0
\end{gathered}
$$

or, via $U_{n}^{\prime}=P^{-1 / 2} U_{n}$, in the form

$$
\begin{aligned}
& \left\{\frac{d^{2}}{d P^{2}}+\frac{(1 / 4)-m^{2}}{P^{2}}+\frac{2 M E_{n, m}}{((\eta / 2)+(q B / 2))^{2}\left(1+\beta P^{2}\right)^{2}}\right. \\
& \quad-\frac{\beta}{1+\beta P^{2}}-\frac{(1+(q B \theta / 4))^{2} P^{2}}{((\eta / 2)+(q B / 2))^{2}\left(1+\beta P^{2}\right)^{2}} \\
& \left.\quad+\frac{2 \beta P}{1+\beta P^{2}} \frac{d}{d P}+\frac{(1+(q B \theta / 4)) m}{((\eta / 2)+(q B / 2))\left(1+\beta P^{2}\right)}\right\} U_{n}=0 .
\end{aligned}
$$

A transformation of the form $p=(1 / \sqrt{\beta}) \operatorname{tg}((x / 2)+$ $(\pi / 4))$ brings (12) into

$$
\begin{aligned}
& \left\{\frac{d^{2}}{d x^{2}}+\frac{\left((1 / 4)-m^{2}\right)\left(1+\operatorname{tg}^{2}((x / 2)+(\pi / 4))\right)^{2}}{4 \operatorname{tg}^{2}((x / 2)+(\pi / 4))}\right. \\
& \quad+\frac{2 M E_{n, m}}{4 \beta((\eta / 2)+(q B / 2))^{2}}-\frac{\left(1+\operatorname{tg}^{2}((x / 2)+(\pi / 4))\right)}{4} \\
& \quad-\frac{(1+(q B \theta / 4))^{2} \operatorname{tg}^{2}((x / 2)+(\pi / 4))}{4 \beta^{2}((\eta / 2)+(q B / 2))^{2}}, \\
& \left.+\frac{\left(1+\operatorname{tg}^{2}((x / 2)+(\pi / 4))\right)(1+(q B \theta / 4)) m}{4 \beta((\eta / 2)+(q B / 2))}\right\} U_{n}=0 .
\end{aligned}
$$


By simplifying (13), we obtain the following equation:

$$
\begin{aligned}
& \left\{\frac{d^{2}}{d x^{2}}+\frac{\left((1 / 4)-m^{2}\right)}{4 \sin ^{2}((x / 2)+(\pi / 4)) \cos ^{2}((x / 2)+(\pi / 4))}\right. \\
& +\frac{2 M E_{n, m}}{4 \beta((\eta / 2)+(q B / 2))^{2}}-\frac{1}{4 \cos ^{2}((x / 2)+(\pi / 4))} \\
& -\frac{(1+(q B \theta / 4))^{2} \sin ^{2}((x / 2)+(\pi / 4))}{4 \beta^{2}((\eta / 2)+(q B / 2))^{2} \cos ^{2}((x / 2)+(\pi / 4))} \\
& \left.+\frac{(1+(q B \theta / 4)) m}{4 \beta((\eta / 2)+(q B / 2)) \cos ^{2}((x / 2)+(\pi / 4))}\right\} U_{n}=0,
\end{aligned}
$$

which, via the change of variable $z=\cos ^{2}((x / 2)+(\pi / 4))$, takes the more familiar form

$$
\begin{aligned}
& \frac{d^{2} U_{n}(z)}{d z^{2}}+\frac{1 / 2-z}{z(1-z)} \frac{d U_{n}(z)}{d z}+\frac{1}{z^{2}(1-z)^{2}} \\
& \quad \times\left\{\alpha_{1} z^{2}+\alpha_{2} z+\alpha_{3}\right\} U_{n}(z)=0,
\end{aligned}
$$

with

$$
\begin{aligned}
\alpha_{1}= & \frac{2 M E_{n, m}}{4 \beta((\eta / 2)+(q B / 2))^{2}}+\frac{(1+(q B \theta / 4))^{2}}{4 \beta^{2}((\eta / 2)+(q B / 2))^{2}}, \\
\alpha_{2}= & \frac{1}{4}+\frac{2 M E_{n, m}}{4 \beta((\eta / 2)+(q B / 2))^{2}}+\frac{(1+(q B \theta / 4))^{2}}{2 \beta^{2}((\eta / 2)+(q B / 2))^{2}} \\
& -\frac{m(1+(q B \theta / 4))}{4 \beta((\eta / 2)+(q B / 2))}, \\
\alpha_{3}= & -\frac{1}{16}+\frac{1}{4}+\frac{m^{2}}{4}+\frac{(1+(q B \theta / 4))^{2}}{4 \beta^{2}((\eta / 2)+(q B / 2))^{2}} \\
& -\frac{m(1+(q B \theta / 4))}{4 \beta((\eta / 2)+(q B / 2))} .
\end{aligned}
$$

Equation (15) possesses the eigenfunctions

$$
\begin{aligned}
U_{n}(z)= & z^{(1 / 4)+\sqrt{(1 / 16)+\alpha_{3}}}(1-z)^{(1 / 4)+\sqrt{(1 / 16)+\alpha_{1}+\alpha_{3}-\alpha_{2}}} \\
& \times P_{n}^{\left(2 \sqrt{(1 / 16)+\alpha_{3}}, 2 \sqrt{(1 / 16)+\alpha_{1}+\alpha_{3}-\alpha_{2}}\right)}(1-2 z),
\end{aligned}
$$

and its energy eigenvalues can be derived from

$$
\begin{aligned}
n(n+1) & +(2 n+1) \\
& \times\left(\sqrt{\frac{1}{16}+\alpha_{1}+\alpha_{3}-\alpha_{2}}+\sqrt{\frac{1}{16}+\alpha_{3}}\right)-\alpha_{2} \\
& +\frac{3}{8}+2 \alpha_{3}+2 \sqrt{\left(\frac{1}{16}+\alpha_{3}\right)\left(\frac{1}{16}+\alpha_{1}+\alpha_{3}-\alpha_{2}\right)} \\
= & 0,
\end{aligned}
$$

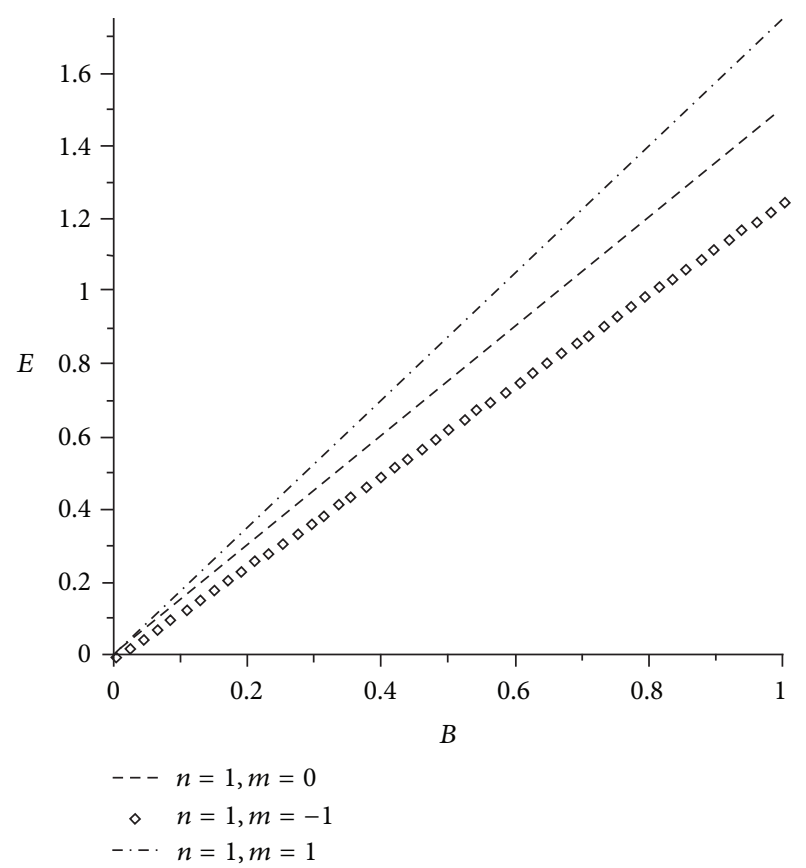

Figure 1: The energy eigenvalues versus $B(\theta=0.000003, \eta=$ $0.000001, M=1)$.

which yields

$$
\begin{aligned}
& E_{n, m} \\
& =\left[\frac{\beta(\eta+q B)^{2} n(n+1)}{2 M}\right. \\
& \quad+\frac{\beta(\eta+q B)^{2} m(2 n+1)}{4 M}+\frac{(\eta+q B)^{2}(2 n+1+m)}{2 M} \\
& \quad \times \sqrt{\beta^{2}\left(\frac{1+m^{2}}{4}\right)+\frac{(1+(q B \theta / 4))^{2}}{(\eta+q B)^{2}}-\frac{\beta m(1+(q B \theta / 4))}{2(\eta+q B)}} \\
& \left.\quad+\frac{\beta(\eta+q B)^{2}\left(1+m^{2}\right)}{4 M}-\frac{m(1+(q B \theta / 4))(\eta+q B)}{4 M}\right] .
\end{aligned}
$$

\section{Special Cases}

Let us now check the special cases. First, when the minimal length is absent, that is, $\beta=0$, the energy relation (19) reduces to

$$
\begin{gathered}
E_{n, m}=\left[\frac{(\eta+q B)(2 n+1+m)(1+(q B \theta / 4))}{2 M}\right. \\
\left.-\frac{m(1+(q B \theta / 4))(\eta+q B)}{4 M}\right] .
\end{gathered}
$$

The energy has plotted the energy eigenvalues versus $B$ in Figure 1. The energy relation in the special cases of $\theta=0$ and 


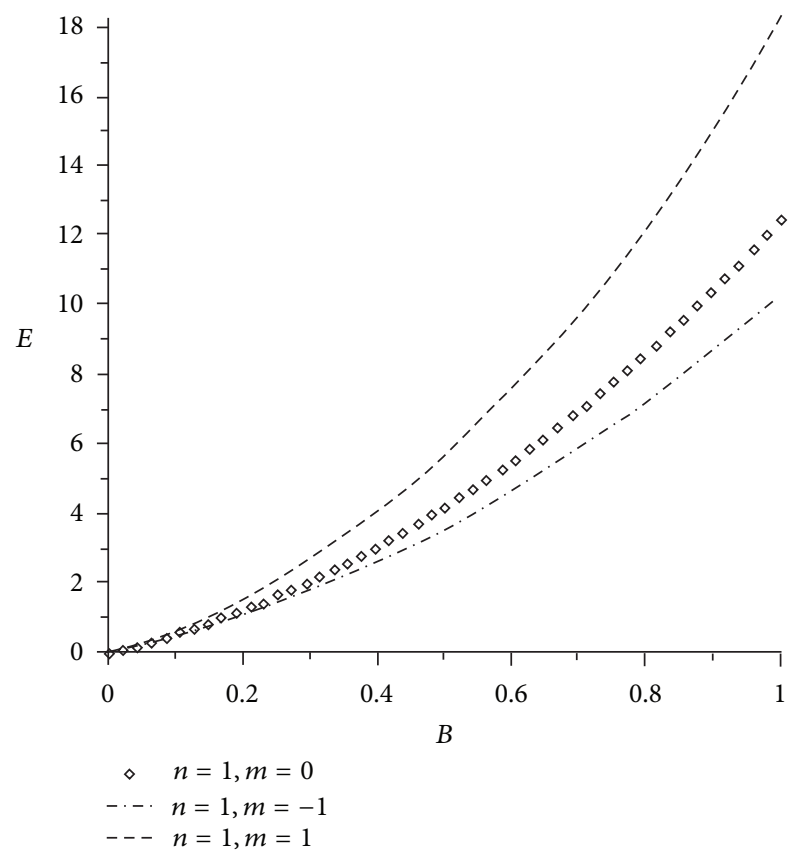

FIgURE 2: The energy eigenvalues versus $B(\beta=1.5, \eta=$ $0.000001, M=0.3)$.

$\eta=0$, that is, for vanishing NCPS parameters, respectively, yields

$$
\begin{aligned}
& E_{n, m}=\left[\frac{\beta(\eta+q B)^{2} n(n+1)}{2 M}+\frac{\beta(\eta+q B)^{2} m(2 n+1)}{4 M}\right. \\
& +\frac{(\eta+q B)^{2}(2 n+1+m)}{2 M} \\
& \times \sqrt{\beta^{2}\left(\frac{1+m^{2}}{4}\right)+\frac{1}{(\eta+q B)^{2}}-\frac{\beta m}{2(\eta+q B)}} \\
& \left.+\frac{\beta(\eta+q B)^{2}\left(1+m^{2}\right)}{4 M}-\frac{m(\eta+q B)}{4 M}\right], \\
& E_{n, m}=\left[\frac{2 \beta q^{2} B^{2} n(n+1)+\beta q^{2} B^{2} m(2 n+1)}{4 M}\right. \\
& +\frac{\beta q^{2} B^{2}\left(1+m^{2}\right)-m q B(1+(q B \theta / 4))}{4 M} \\
& +\frac{q^{2} B^{2}(2 n+1+m)}{2 M} \\
& \times\left(\beta^{2}\left(\frac{1+m^{2}}{4}\right)+\frac{(1+(B q \theta / 4))^{2}}{q^{2} B^{2}}\right. \\
& \left.\left.-\frac{\beta m(1+(q B \theta / 4))}{2 q B}\right)^{1 / 2}\right] \text {. }
\end{aligned}
$$

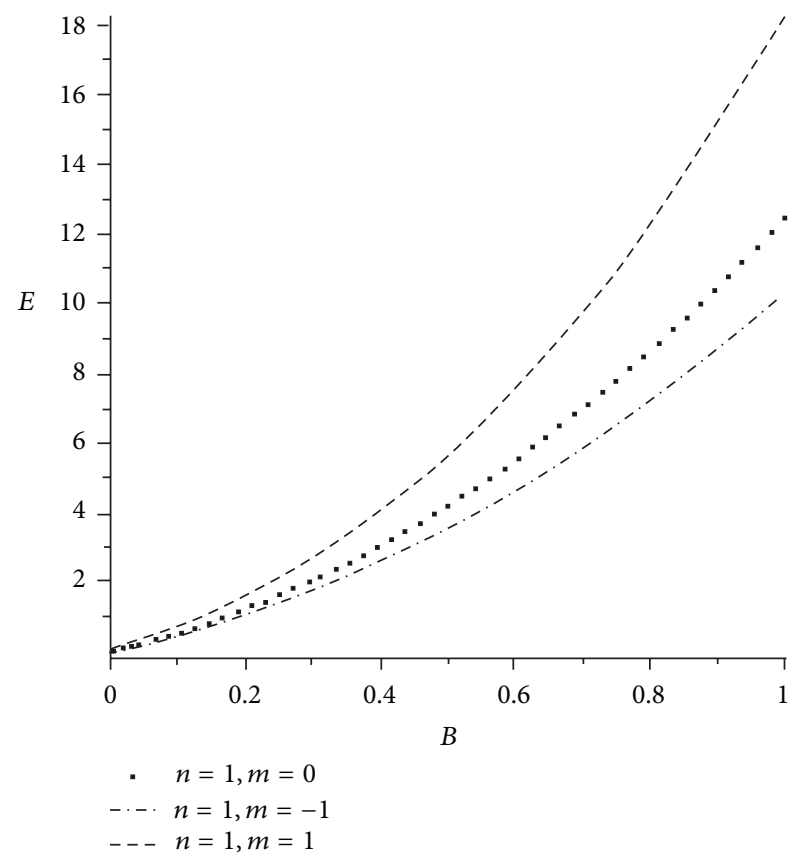

Figure 3: The energy eigenvalues versus $B(\beta=1.5, \theta=$ $0.000001, M=0.3)$.

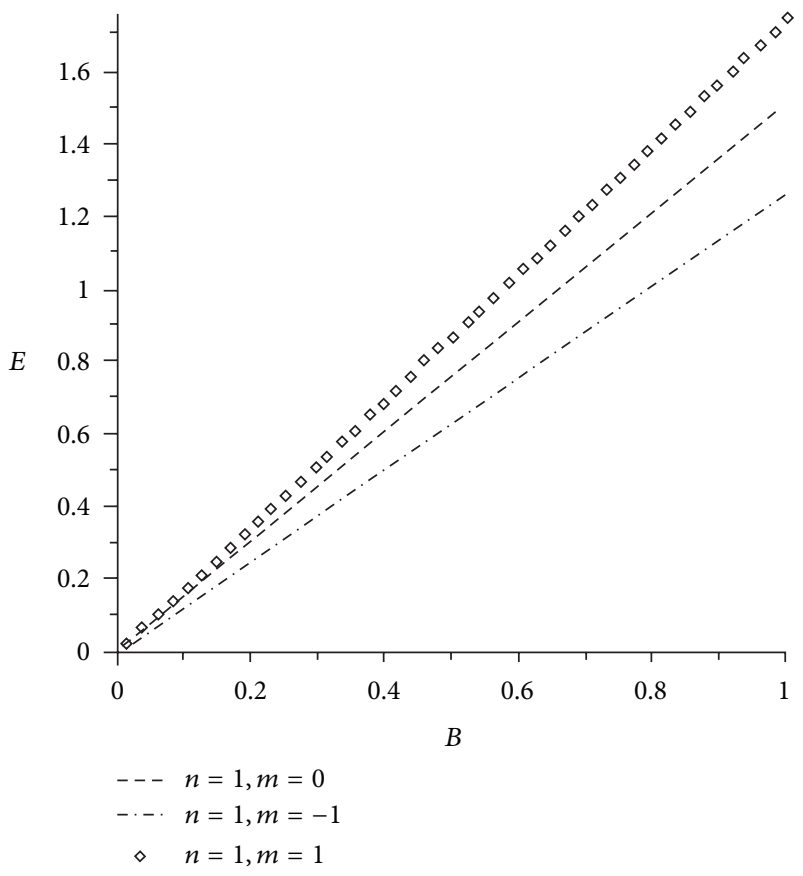

FIgURE 4: The energy eigenvalues versus $B(M=1)$.

We have depicted in Figures 2 and 3 the energy versus $B$. Finally, when both the noncommutative and minimal length parameters are absent, that is, when $\beta=\theta=\eta=0$, and also $\left(q^{2} B^{2} / 8 M\right) \rightarrow 1 / 2 M \omega^{2}$, the energy spectrum takes the form

$$
E_{n, m}=\omega_{0}(2 n+1+m) .
$$


This is the known form of energy in [29-31]. In Figure 4, we have shown the energy values versus $B$.

\section{Conclusion}

In this study, we considered the Schrödinger equation incorporated with two recent trends in the field: the noncommutative formulation and the minimal length uncertainty relation. After two novel transformations, we transformed the equation into a familiar form and reported the solutions in terms of the Jacobi polynomials. Our results, in the special cases of the engaged parameters, yield the previous results.

\section{Conflict of Interests}

The authors declare that there is no conflict of interests regarding the publication of this paper.

\section{Acknowledgment}

The authors wish to give their sincere gratitude to the referees for their technical comments on the paper.

\section{References}

[1] L. I. Schiff, Quantum Mechanics, McGraw-Hill, New York, NY, USA, 3rd edition, 1955.

[2] R. L. Liboff, Introductory Quantum Mechanics, Addison Wesley, San Francisco, Calif, USA, 4th edition, 2003.

[3] M. M. Nieto, "Hydrogen atom and relativistic pi-mesic atom in $\mathrm{N}$-space dimensions," American Journal of Physics, vol. 47, no. 12, pp. 1067-1072, 1979.

[4] H. S. Snyder, "Quantized space-time," Physical Review, vol. 71, no. 1, pp. 38-41, 1947.

[5] T. Banks, W. Fischler, S. H. Shenker, and L. Susskind, " $M$ theory as a matrix model: a conjecture," Physical Review D, vol. 55, no. 8, pp. 5112-5128, 1997.

[6] N. Seiberg and E. Witten, "String theory and noncommutative geometry," Journal of High Energy Physics, vol. 1999, no. 9, article 32, 1999.

[7] F. Lizzi, G. Mangano, G. Miele, and M. Peloso, "Cosmological perturbations and short distance physics from noncommutative geometry," Journal of High Energy Physics, vol. 2002, no. 6, article 49, 2002.

[8] R. Brandenberger and P.-M. Ho, "Noncommutative spacetime, stringy spacetime uncertainty principle, and density fluctuations," Physical Review D, vol. 66, no. 2, Article ID 023517, 10 pages, 2002.

[9] B. Vakili, N. Khosravi, and H. R. Sepangi, "Bianchi spacetimes in noncommutative phase space," Classical and Quantum Gravity, vol. 24, no. 4, pp. 931-949, 2007.

[10] S. Minwalla, M. van Raamsdonk, and N. Seiberg, "Noncommutative perturbative dynamics," Journal of High Energy Physics, vol. 2000, no. 2, article 20, 2000.

[11] S. M. Carroll, J. A. Harvey, V. A. Kostelecký, C. D. Lane, and T. Okamoto, "Noncommutative field theory and Lorentz violation," Physical Review Letters, vol. 87, no. 14, Article ID 141601, 4 pages, 2001.

[12] R. J. Szabo, "Quantum field theory on noncommutative spaces," Physics Reports, vol. 378, no. 4, pp. 207-299, 2003.
[13] M. R. Douglas and N. A. Nekrasov, "Noncommutative field theory," Reviews of Modern Physics, vol. 73, no. 4, pp. 977-1029, 2001.

[14] J. Gamboa, M. Loewe, and J. C. Rojas, "Noncommutative quantum mechanics," Physical Review D, vol. 64, no. 6, Article ID 067901, 3 pages, 2001.

[15] M. Przanowski and J. Tosiek, "The Weyl-Wigner-Moyal formalism. III. The generalized Moyal product in the curved phase space," Acta Physica Polonica B, vol. 30, no. 2, p. 179, 1999.

[16] B. Mirza and M. Mohadesi, "The Klein-Gordon and the Dirac oscillators in a noncommutative space," Communications in Theoretical Physics, vol. 42, no. 5, pp. 664-668, 2004.

[17] D. J. Gross and P. F. Mende, "String theory beyond the Planck scale," Nuclear Physics B, vol. 303, no. 3, pp. 407-454, 1988.

[18] D. Amati, M. Ciafaloni, and G. Veneziano, "Can spacetime be probed below the string size?” Physics Letters B, vol. 216, no. 1-2, pp. 41-47, 1989.

[19] M. Maggiore, "The algebraic structure of the generalized uncertainty principle," Physics Letters B, vol. 319, no. 1-3, pp. 83-86, 1993.

[20] G. Amelino-Camelia, N. E. Mavromatos, J. Ellis, and D. V. Nanopoulos, "On the space-time uncertainty relations of Liouville strings and D-branes," Modern Physics Letters A, vol. 12, no. 27, pp. 2029-2035, 1997.

[21] F. Brau and F. Buisseret, "Minimal length uncertainty relation and gravitational quantum well," Physical Review D, vol. 74, no. 3, Article ID 036002, 5 pages, 2006.

[22] H. Hassanabadi, S. Zarrinkamar, and E. Maghsoodi, "Scattering states of Woods-Saxon interaction in minimal length quantum mechanics," Physics Letters B, vol. 718, no. 2, pp. 678-682, 2012.

[23] S. Das and E. C. Vagenas, "Universality of quantum gravity corrections," Physical Review Letters, vol. 101, no. 22, Article ID 221301, 4 pages, 2008.

[24] A. F. Ali, S. Das, and E. C. Vagenas, "Proposal for testing quantum gravity in the lab," Physical Review D, vol. 84, no. 4, Article ID 044013, 10 pages, 2011.

[25] H. Hassanabadi, Z. Molaee, and S. Zarrinkamar, "DKP oscillator in the presence of magnetic field in $(1+2)$-dimensions for spin-zero and spin-one particles in noncommutative phase space "' The European Physical Journal C, vol. 72, article 2217, 2012.

[26] O. Bertolami, J. G. Rosa, C. M. L. de Aragão, P. Castorina, and D. Zappalà, "Scaling of variables and the relation between noncommutative parameters in noncommutative quantum mechanics," Modern Physics Letters A, vol. 21, no. 10, pp. 795802, 2006.

[27] O. Bertolami, J . G. Rosa, C. M. L. de Aragão, P. Castorina, and D. Zappalà, "Noncommutative gravitational quantum well," Physical Review D, vol. 72, no. 2, Article ID 025010, 9 pages, 2005.

[28] L. Menculini, O. Panella, and P. Roy, "Exact solutions of the (2 +1 ) dimensional Dirac equation in a constant magnetic field in the presence of a minimal length," Physical Review D, vol. 87, no. 6, Article ID 065017, 10 pages, 2013.

[29] A. Hatzinikitas and I. Smyrnakis, "The noncommutative harmonic oscillator in more than one dimension," Journal of Mathematical Physics, vol. 43, no. 1, pp. 113-125, 2002.

[30] D. Karabali, V. P. Nair, and A. P. Polychronakos, "Spectrum of Schrödinger field in a noncommutative magnetic monopole," Nuclear Physics B, vol. 627, no. 3, pp. 565-579, 2002. 
[31] A. Smailagic and E. Spallucci, "Isotropic representation of the noncommutative 2D harmonic oscillator," Physical Review D, vol. 65, no. 10, Article ID 107701, 4 pages, 2002. 

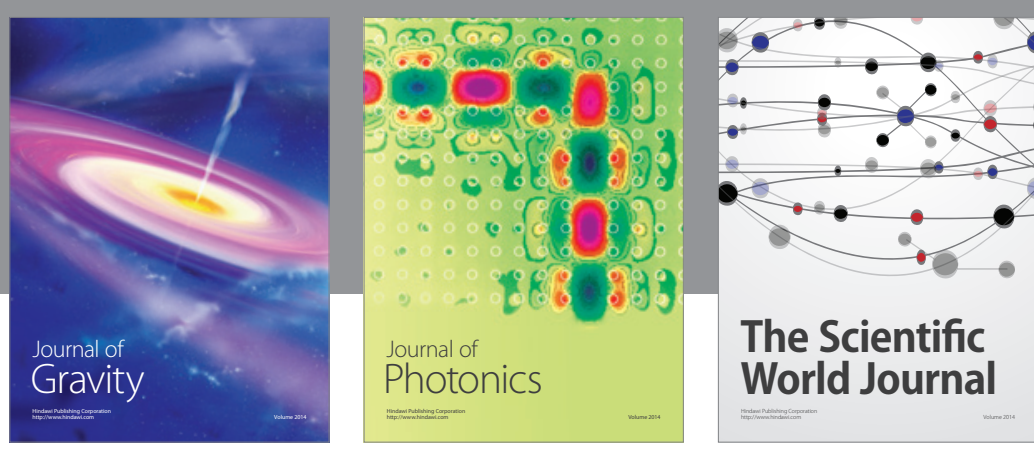

The Scientific World Journal
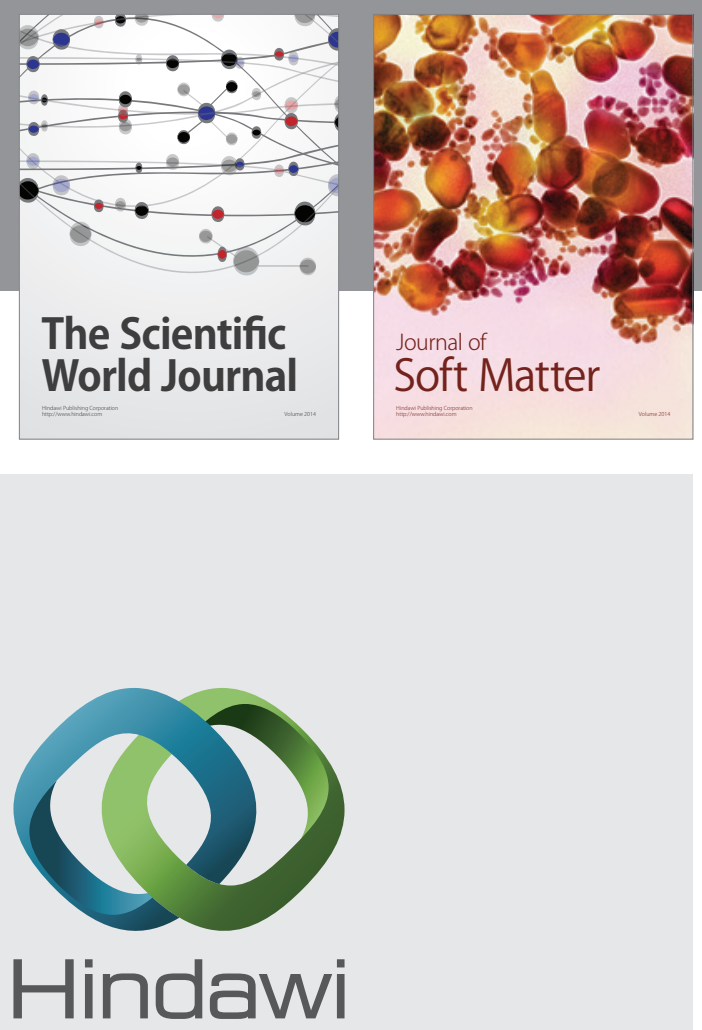

Submit your manuscripts at

http://www.hindawi.com

nternational Journal of

Statistical Mechanics
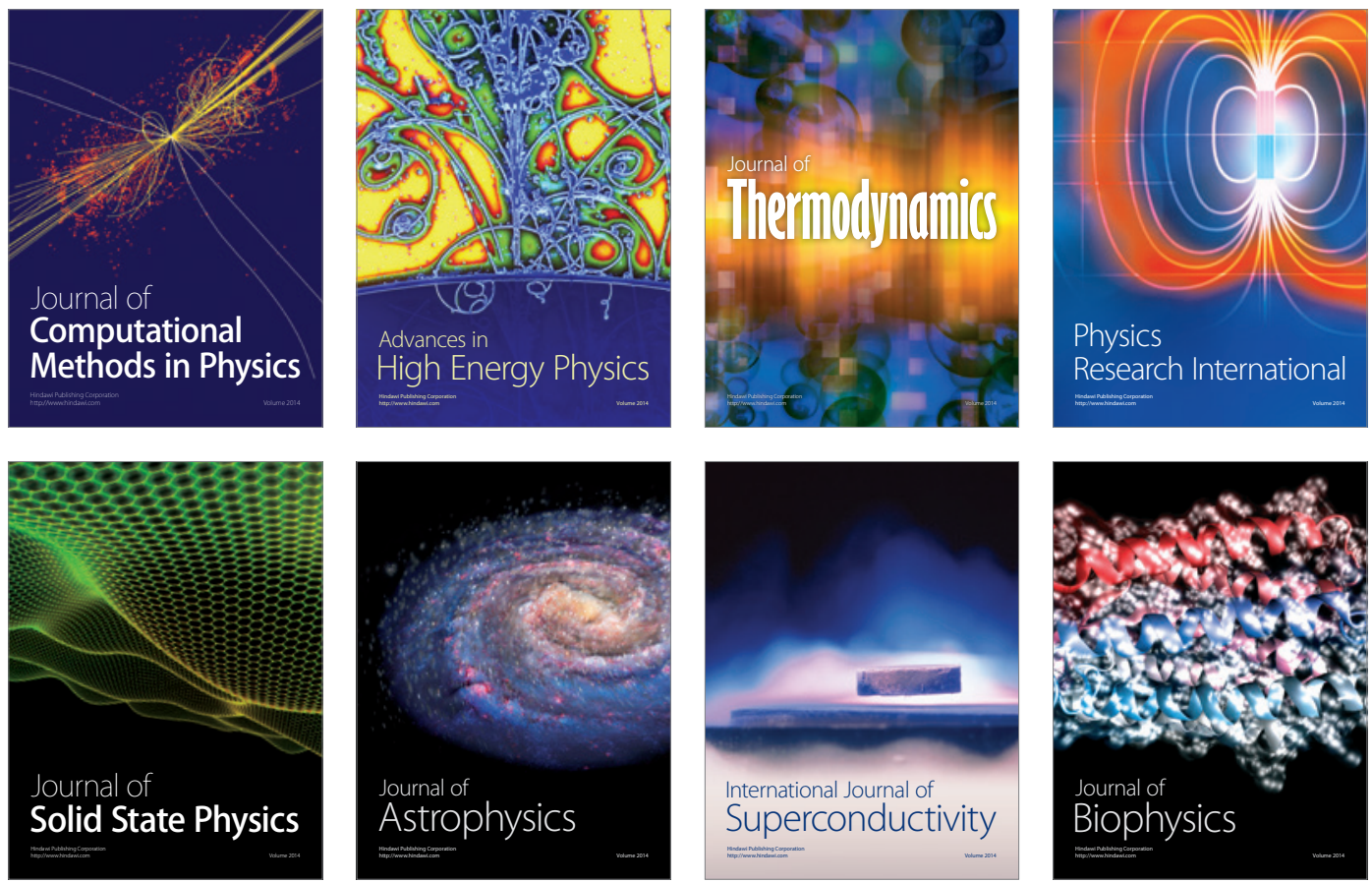
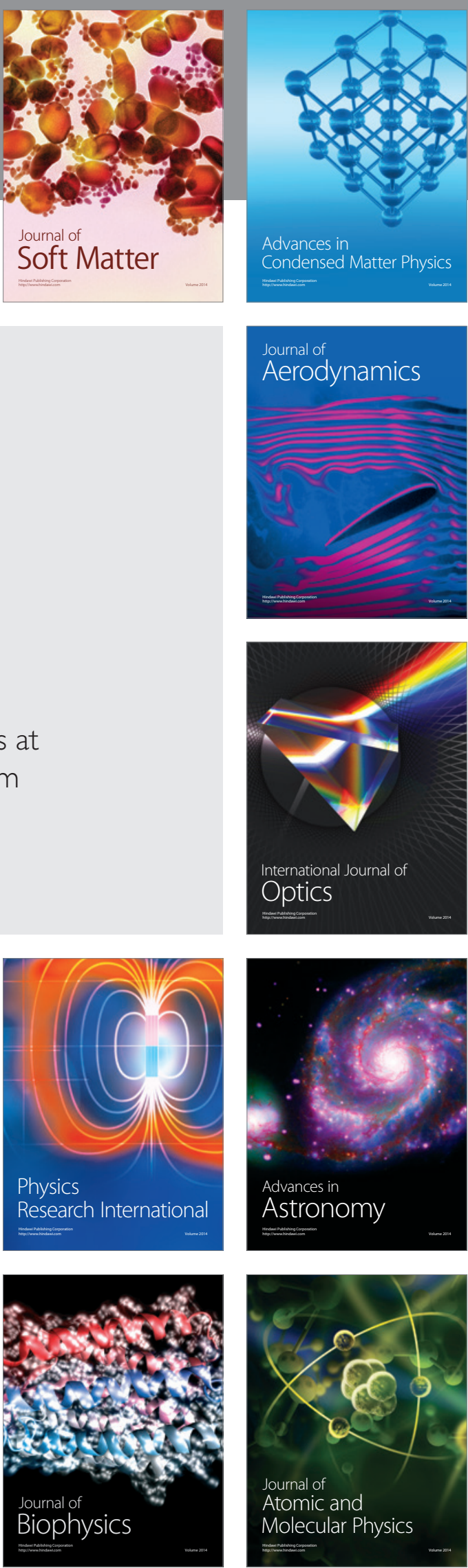